\title{
An Extragenital Colonic Salpingiosis
}

\author{
Valeria D'Ovidio $^{1}$, Daniela Maggi ${ }^{2}$, Giovanni Bruno $^{1}$, Stefano Fratoni $^{3}$, Marco Guazzaroni ${ }^{1}$ \\ 1) Gastrointestinal Endoscopy Unit, S.Eugenio Hospital, Rome; 2) Gastrointestinal Unit, Policlinico Umberto I, Rome; 3) Pathology Unit, \\ S.Eugenio Hospital, Rome, Italy
}
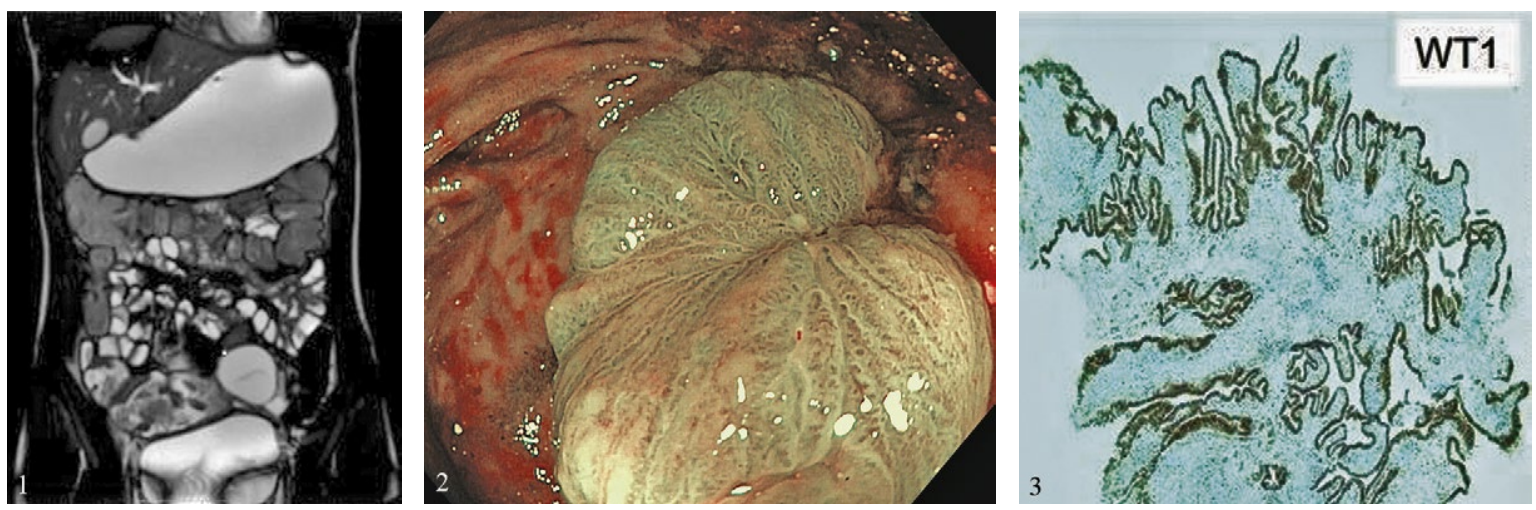

A 22-year-old female was referred for recurrent abdominal pain in the right-lower quadrant without fever or weight loss. She denied smoking habits and reported a family history for Crohn's disease. Laboratory investigation revealed increased fecal calprotectine $(125 \mathrm{mg} / \mathrm{dl})$ and C-reactive protein $(4 \mathrm{mg} /$ dl) values. Magnetic resonance imaging (MRI) showed an abscess in the left ovary (Fig. 1). Ileo-colonoscopy revealed a distorted mucosa, proximal to the appendix, with a superficial neoplastic lesion (10 $\mathrm{mm}$ diameter). The lesion was irregular shaped with a central depression and a glandular pattern, being better characterized by virtual chromo-endoscopy (narrow band imaging) (0-I s Paris type) (Fig. 2).

The histology revealed the fallopian tube's fimbriated end. On immune-histochemical evaluation, the epithelium strongly expressed nuclear staining for estrogen, progesterone and Wilms' tumor protein 1 (WT1) receptors, consistent with endosalpingiosis (Fig. 3). Colonic endosalpingiosis with inverted appendix was diagnosed. The intussusception was due to an isolated appendicular localization of endosalpingiosis without other localization visible on MRI. A multidisciplinary team (gynecologist, gastroenterologist, surgeon) decided laparoscopic resection of focal endosalpingiosis. One week after surgery she was discharged uneventfully without any recurrence in the 12 months of follow-up.

Endosalpingiosis is seen exclusively in women of reproductive age usually involving the peritoneum and the retroperitoneal lymph-nodes. Extra-genital endosalpingiosis is a rare condition reported only once as chronic pelvic pain, likely related to coexisting endometriosis $[1,2]$. The histogenesis is controversial; following a newer hypothesis of müllerianosis, endosalpingiosis is thought to originate from preexisting müllerian rests [3]. The endoscopic findings are not typical. To our knowledge, the endoscopic appearance of endosalpingiosis has never been reported in the literature. Histopathologic evaluation provided definitive diagnosis especially in our case where endoscopic appearance can mimic an adenoma.

Corresponding author: Valeria D’Ovidio; valeria.dovidio@aslroma2.it

Conflicts of interest: None to declare.

\section{REFERENCES}

1. Davies SA, Maclin VM. Endosalpingosis as a cause of chronic pelvic pain. Am J Obstet Gynecol 1991;164:495-496. doi:10.1016/s00029378(11)80007-4

2. Heinig J, Gottschalk I, Cirkel U, Diallo R. Endosalpingiosis - an underestimated cause of chronic pelvic pain or an accidental finding? A retrospective study of 16 cases. Eur J Obstet Gynecol Reprod Biol 2002;103:75-78. doi:10.1016/s0301-2115(02)00020-9

3. Koren J, Mensikova J, Mukensnabl P, Zamecnik M. Müllerianosis of the urinary bladder: report of a case with suggested metaplastic origin. Virchows Arch 2006;449:268-271. doi:10.1007/s00428-006-0153-1 